\title{
Éditorial
}

\section{Les créneaux territoriaux}

La politique territoriale du gouvernement du Québec est à l'heure des créneaux. Certains les désignent sous l'appellation de filières de production. D'autres préfèrent utiliser l'expression "clusters» ou grappes industrielles. D'autres encore considèrent qu'il s'agit de systèmes de production localisée, en référence au vocabulaire utilisé par l'OCDE, l'Organisation de coopération et de développement économique. Toutes ces expressions ne désignent évidemment pas tout à fait le même phénomène, dans ses variantes sectorielles et territoriales. Et chaque expression possède, selon les auteurs, différentes définitions plus ou moins distinctes. D’une manière générale, le contenu conceptuel associé au «créneau territorial» concerne des entreprises qui, naturellement, se localisent sur un même territoire afin de mieux s'intégrer ensemble dans un processus de production fragmenté en plusieurs unités et segments. L'idée d'une politique publique en regard des créneaux vient de la volonté gouvernementale de soutenir cette naturelle agglomération d'entreprises, en fonction de ses effets positifs sur la production de richesses et sur la diffusion du développement sur le vaste espace national.

À cet effet au Québec, Montréal possède déjà ses Cités du multimédia et de l'électronique alors qu'une Cité de l'optique - photonique prend de plus en plus assise à Québec. Le concept de technopôle est appliqué sur les créneaux de l'agro-alimentaire (Saint-Hyacinthe), de l'aérospatial (Laval) et du maritime (Rimouski). Au Saguenay et en Basse-Mauricie, la notion de Vallée sert en outre ce même enjeu de consolidation d'un créneau spécifique. Et désormais, cette approche d'intervention gouvernementale sur la base des créneaux cherche sa généralisation à l'ensemble du Québec. Sont ainsi actuellement identifiés trois, quatre ou cinq créneaux par régions, notamment ceux de l'éolien en Gaspésie, de la phytogénétique sur la Côte-Nord, de la tourbe dans le Bas-Saint-Laurent, du récréotourisme dans les Laurentides, des technomines souterraines en Abitibi. Il en existe beaucoup d'autres aussi, comme la forêt et le textile qui s'étendent sur de vastes zones suprarégionales ou comme ledit tertiaire - moteur qui structure les pôles régionaux. Sans compter le potentiel que renferme les petits créneaux tels que les produits de la mer dans l'est ou de l'érable dans le sud, les petits fruits sauvages plus au nord, l'agneau dans le Bas-du- fleuve, le bio-alimentaire dans des zones ici et là, les bleuets au Lac-Saint-Jean, le maïs en Montérégie, les pommes de terre au Saguenay, le veau dans Charlevoix, etc.

Avant de se traduire sous la forme d'un programme gouvernemental spécifique au Québec, mais aussi en Allemagne, en Suède et en France, cette approche «créneau» pour ancrer la politique de développement territorial correspond d'abord à une réalité empirique et ensuite à un cadre théorique. L'origine de l'observation et de la modélisation des créneaux remonte au début du siècle dans les travaux sur les districts industriels de l'économiste anglais Alfred Marshall. N'ayant que peu attiré les projecteurs de la science pendant les décennies suivantes, la présence de «clusters» fut clairement remise en évidence par le mouvement de recherche sur les échanges interindustriels, dans les années 1950 et 1960. Pendant les années 1970, le Professeur Stan Zcamanski de l'Université de Cornell fut le premier à appliquer systématiquement cette approche dans l'est du Canada, pour le compte du gouvernement fédéral. Au début des années 1980, les nouveaux districts industriels en Italie, les zones à forte valeur ajoutée aux États-Unis ainsi que les concentrations technologiques localisées ici et là partout sur la planète, ont fortement marqué la théorie $d u$ développement territorial. Ces grappes d'entreprises furent en réalité propulsées au rang de "territoires à succès», de "milieux innovateurs", de «régions gagnantes» et désormais de «communautés apprenantes». En se basant sur le corpus théorique déjà acquis, les analystes scientifiques contemporains ont modélisé ces lieux et ces zones spécifiques par l'entremise notamment de la perspective systémique afin de saisir et comprendre le jeu relationnel des divers acteurs dans l'organisation globale de la branche industrielle (ou secteur) présente sur un territoire donné. L'approche créneau fut alors consacrée pour sa capacité d'effectuer la liaison analytique entre l'organisation industrielle et l'organisation territoriale.

Au Québec, l'approche de développement territorial s'appuyant sur les créneaux fut spécifiquement proposée à la population lors du Rendez-vous des Régions en novembre 2002. Selon notre lecture, l'intérêt de cette approche pour le Québec non métropolitain s'articule autour de quatre enjeux que voici. 
Le créneau territorial est d'abord une nouvelle méthode d'observation et d'analyse des filières de production appliquée à une échelle relativement fine. Elle est plaquée actuellement sur d'autres méthodes similaires plus anciennes, comme celle des grappes industrielles qui fut très populaire au début des années 1990 ou celle des divers secteurs et sous-secteurs de production qui a fortement marqué l'élaboration de la première véritable politique industrielle du Québec au tout début des années 1980. On pourrait même, si désiré, remonter au principe des «industries industrialisantes» qui a largement crédibilisé la volonté de promotion des pôles de développement identifiés par le MIC (Ministère de l'industrie et du commerce) au milieu des années 1960. En contexte contemporain, cette méthode "créneau» nous permet de mieux comprendre trois dimensions centrales des économies territoriales. Sans épiloguer longuement sur celles-ci dans cet éditorial, il s'agit à titre indicatif:

- des interrelations entre les divers segments dans la chaîne amont - aval de valorisation successive à partir de la matière première vers le produit final à commercialiser;

- du champ concurrentiel (fournisseurs, clients, leaders, substituts...) dans ses forces, faiblesses, menaces, contraintes et opportunités;

- de l'évolution par cycles, par itération, par cumul, par étapes de l'ensemble de la structure sectorielle de production.

L'approche par les créneaux offre ensuite au Québec de nouveaux champs potentiels d'interaction pour animer et soutenir les relations, le réseautage, la concertation, la coopération, les partenariats et les alliances. Ceci est d'autant plus vrai puisque cette approche "créneau» interpelle actuellement, de premier chef, le secteur privé qui est une source constante de nouveaux leaderships. Ayant leur territorialité propre, généralement différente des aires officielles de gestion publique, les créneaux à soutenir par la politique publique seront inévitablement confrontés aux frontières institutionnalisées et bien gardées des municipalités, des MRC (municipalités régionales de comté) et des régions administratives. Ainsi, l'utilisation de la territorialité réelle des

Paul Prévost

Université de Sherbrooke créneaux représente actuellement un défi considérable à relever. S'ils réussissent à prendre la place qui leur revient cependant, la dynamique collective d'apprentissage au développement qui sera stimulée par l'approche "créneau» renouvellera certes d'une manière bénéfique, la concertation régionale qui s'est considérablement cristallisée depuis la belle époque des Conférences socio-économiques. De nouveaux mécanismes d'interaction et de relations seront alors expérimentés, inspirés notamment par l'entremise des technologies d'information et de communication (TIC).

Comme troisième enjeu des créneaux territoriaux au Québec, soulignons la capacité de renouvellement des mesures publiques de soutien au développement local et régional, associée à cette nouvelle approche. Par l'entremise d'un diagnostic approprié sur l'état d'avancement de chaque créneau (filière potentielle, induite ou avérée; présence de filière, limitée ou manifeste; consolidation partielle ou étendue de la filière; excellence tangible ou supérieure; intégration parfaite de la filière sous la forme de district) et de son positionnement concurrentiel (technologies; coûts; innovation; etc.), tout en se référant au modèle idéal de filière industrielle tel qu'illustré par les cas de maturité, il devient alors possible de déduire des points d'appui pertinents (segments faibles; goulots d'étranglement; expertise; qualité des transactions; entrepreneuriat; $R \& D$; etc.) pour les interventions publiques.

Finalement, l'approche par les créneaux territoriaux que Québec met de l'avant implique directement un nouveau joueur très important dans la politique publique de développement territorial, soit la Société Générale de Financement. Il ne s'agit pas d'une mince affaire puisque cet organisme s'avère, comme on le sait, le principal instrument de la politique industrielle du Québec. L'expertise et les moyens de la SGF permettront sans aucun doute de faire progresser les différents créneaux territoriaux vers une plus grande maturité économique et ainsi vers une plus grande valeur ajoutée aux ressources québécoises. Dans une optique de création de richesse à redistribuer, il s'agit là d'un défi important à relever par la SGF et par la politique territoriale du Québec.

Bonne lecture de ce deuxième numéro du volume 12.

Marc-Urbain Proulo

Université du Québec à Chicoutimi 